\title{
Current Therapies for Lupus Nephritis in an Ethnically Heterogeneous Cohort
}

\author{
TANIA L. RIVERA, H. MICHAEL BELMONT, SEEMA MALANI, MELISSA LATORRE, LAURI BENTON, \\ JOSEPH WEISSTUCH, LAURA BARISONI, CHUNG-E TSENG, PETER M. IZMIRLY, JILL P. BUYON, \\ and ANCA D. ASKANASE
}

\begin{abstract}
Objective. To evaluate responses to mycophenolate mofetil (MMF) and intravenous cyclophosphamide (CYC) in lupus nephritis in a multiethnic population.

Methods. This was a retrospective study of all patients with systemic lupus erythematosus (SLE) that underwent kidney biopsy at New York University Medical Center. Patients with followup of at least 6 months were included. Clinical response was defined as complete (return to $\pm 10 \%$ of normal) or partial (improvement of $50 \%$ in abnormal renal measurements).

Results. Ninty-nine patients were included in the study: $86 \%$ females, $86 \%$ non-Caucasian, age 34.2 \pm 1.1 years, $62 \%$ with proliferative nephritis (PN; ISN/RPS-III and IV), and $32 \%$ with membranous nephritis (MN; ISN/RPS-V). Of the 70 patients with PN, 37 were treated with CYC and 33 with MMF. The baseline characteristics of the 2 treatment groups were different in the incidence of ISN/RPS-IV, values of serum creatinine and serum albumin, and type of insurance $(p<0.05)$. The response rate was greater in the MMF than in the CYC group (70\% vs 41\%). Responses to MMF were different in Asians (11/11), Caucasians (4/5), African Americans (3/5), and Hispanics (5/11). Responses to CYC had a similar distribution (Asians 6/10, Caucasians 4/5, African Americans 4/9, Hispanics 1/11). In the MN group ( $\mathrm{N}=23)$ responses were similar to the $\mathrm{PN}$ group $(73 \% \mathrm{MMF}$ and $38 \%$ CYC). After adjusting for race, serum creatinine, serum albumin, type of insurance, and class of nephritis, in a logistic regression model, response to MMF was superior to CYC: OR 6.2 (95\% CI 1.9-20.2). Hispanics had worse outcome than Caucasians (OR 0.17). Longterm followup suggested no difference in maintenance with MMF or CYC.

Conclusion. After controlling for the fact that less severe nephritis is preferentially treated with MMF, we found overall that response to MMF was superior to CYC. In this US population, ethnicity was observed to have an influence on response. (First Release Dec 1 2008; J Rheumatol 2009; 36:298-305; doi:10.3899/jrheum.080335)
\end{abstract}

Key Indexing Terms:

CYCLOPHOSPHAMIDE

LUPUS NEPHRITIS

\section{MYCOPHENOLATE MOFETIL HISPANICS}

Lupus nephritis (LN) is a major cause of morbidity and mortality in patients with systemic lupus erythematosus (SLE). Treatment approaches are traditionally considered in 2 phases, induction and maintenance. During induction, high doses of immunosuppressives and cytotoxics are generally admin-

From New York University School of Medicine; Downtown Hospital, New York; SUNY Downstate, Brooklyn; and Columbia University, New York, New York, USA.

Supported in part by an Investigator Initiated Grant from Aspreva Pharmaceuticals.

T.L. Rivera, MD; H. M. Belmont, MD; J. Weisstuch, MD; L. Barisoni, MD; C-E. Tseng, MD; P.M. Izmirly, MD; J.P. Buyon, MD; A.D. Askanase, $M D, M P H$, New York University School of Medicine; S. Malani, MD, Downtown Hospital; M. Latorre, BSE, SUNY Downstate; L. Benton, MD, MPH, Columbia University.

Address reprint requests to Dr. A.D. Askanase, Department of Medicine, Division of Rheumatology, New York University School of Medicine,

560 First Avenue, TCH-407, New York, NY.

E-mail: ardinu@aol.com or anca.askanase@nyumc.org

Accepted for publication September 11, 2008. istered in conjunction with glucocorticoids to reverse immune-mediated inflammatory processes in the anticipation of preventing progressive and irreversible fibrosis. Thereafter, maintenance therapy with lower and presumably safer levels of immunosuppressive medications is intended to assure durability of response and prevent recurrences.

Intravenous cyclophosphamide (CYC; Cytoxan) has been the standard treatment for $\mathrm{LN}$ in the United States since the US National Institutes of Health (NIH) trial publication in 1986, and is used for both induction and maintenance ${ }^{1,2}$. However, renal improvement is often accompanied by cytotoxicity, thus leading to side effects such as bone marrow suppression, gonadal toxicity, hemorrhagic cystitis, herpes zoster, alopecia, and increased risk of cancer. In an effort to avoid these toxicities, azathioprine has been used as an alternative to CYC during maintenance therapy. Moreover, recent studies have shown that mycophenolate mofetil (MMF; CellCept) provides efficacy comparable to CYC for both induction and maintenance with fewer side effects ${ }^{3-7}$. 
MMF is an immunosuppressive agent that suppresses $d e$ novo purine synthesis by selectively inhibiting inosine-5'monophosphate dehydrogenase ${ }^{8}$. Unlike other cell types, lymphocytes are heavily dependent upon this mechanism for proliferation. As a result, MMF is capable of attenuating the proliferation of lymphocytes and subsequent production of antibodies without producing the cytotoxic or mutagenic effects of $\mathrm{CYC}^{9}$.

However, clinical trials from different countries comparing these 2 medications are discordant in their response rates. In the study conducted by Chan, et al, 95\% achieved clinical response (both complete and partial) with MMF and $90 \%$ with $\mathrm{CYC}^{4,5}$. In contrast, in the study by Ginzler, et al, only $52 \%$ responded to MMF and $30 \%$ to $\mathrm{CYC}^{6}$.

The disparity observed between the Chan and Ginzler studies may be attributed to inherent differences in design and patient population. Most recently, Aspreva Pharmaceuticals reported preliminary clinical trial results that showed a $56 \%$ response rate with MMF and 53\% with $\mathrm{CYC}^{10}$.

Finally, except for data from the LUMINA cohort, little is known about disease manifestations in patients of different ethnic backgrounds ${ }^{11}$.

Our study was initiated to evaluate the efficacy of MMF and CYC in inducing responses in a large multiethnic population of patients with $\mathrm{LN}$ treated at an academic institution, and determine the "real-life" experience, since responses to induction with MMF and CYC in everyday clinical practice may be different from clinical trials. The patterns of response and recurrence in patients undergoing maintenance with CYC or MMF over a period of up to 7 years were also compared.

\section{MATERIALS AND METHODS}

Patients and clinical information. This was a single-center retrospective chart review of all SLE patients who underwent a kidney biopsy at the New York University Medical Center (NYUMC) between January 2000 and February 2007. After institutional review board approval of this study, the pathology database was queried to identify all patients whose biopsy indicated a diagnosis of LN. Biopsies were classified (and reclassified) according to the International Society of Nephrology/Renal Pathology Society (ISN/RPS) ${ }^{12}$ as class II (mesangial), class III (focal proliferative), class IV (diffuse proliferative), class V (membranous), and class VI (sclerotic). All available medical records for these patients were reviewed.

The information collected for each patient included full pathology reports, demographics (age, sex, race), documentation of American College of Rheumatology (ACR) criteria for SLE diagnosis, comorbidities, medications, and whether they had a renal transplant or were on hemodialysis. Laboratory values reflecting renal function (blood urea nitrogen, creatinine), urine microscopy (presence of erythrocytes, leukocytes, and casts), and protein excretion (spot protein/creatinine ratio, $24 \mathrm{~h}$ protein, $24 \mathrm{~h}$ protein/creatinine ratio, serum albumin), complement (C3 and $\mathrm{C} 4)$, antidsDNA antibody, and possible side effects of medication were recorded.

Treatment regimens. Induction therapy consisted of either CYC or MMF. CYC was given as monthly pulses according to a protocol of the $\mathrm{NIH}^{13}$. Dosage was modified on the basis of the nadir white blood cell (WBC) count of $\geq 2500$ cells $/ \mathrm{mm}$ per $\mathrm{m}^{2}$ of body-surface area at 10 to 14 days after infusion. MMF was initiated at a dose of $500 \mathrm{mg}$ twice daily, the dose was then increased to $500 \mathrm{mg}$ three times daily at Week 2, and advanced weekly to a maximum dose of $1500 \mathrm{mg}$ twice daily unless the WBC count fell to $<3000 / \mathrm{mm}$ or there was gastrointestinal intolerance, in which case dose was adjusted. Prednisone was administered in conjunction with both medications at $1 \mathrm{mg} / \mathrm{kg}$ for 4 to 8 weeks, and then tapered. Maintenance therapy consisted of CYC pulses administered every 3 months following the same protocol used during induction ${ }^{13}$; the dose was maintained at the level that achieved response during induction, unless toxicities occurred. Alternatively, they were treated with MMF administered at 2-3 g per day up to 2 years. Assignment to either of these groups was at the discretion of the clinicians.

Treatment groups were assigned as follows for induction and maintenance (induction/maintenance): (1) MMF/MMF, (2) CYC/CYC, and (3) CYC/MMF

Definition of outcomes

Induction. Patients were eligible for analysis if they were followed for at least 6 months. Induction response was evaluated 6 months after the initiation of therapy. Patients were classified as complete responders (CR) if they returned to within $10 \%$ of normal values of serum creatinine, proteinuria and urinary sediment; partial responders (PR) if they had an improvement from baseline of $\geq 50 \%$ in all abnormal renal variables without deterioration of any measurements; and nonresponders (NR) if there was improvement in $<3$ variables, or if there was deterioration or no change in the abnormal renal variables.

Maintenance. The primary endpoint was renal flare, defined as a doubling of urine protein excretion; protein/creatinine ratio if the initial ratio was $>0.5$; or a ratio $>1$ if initial value was $<0.5$; and/or a $50 \%$ increase in serum creatinine. The secondary endpoint was renal survival. Chronic renal failure was defined as a sustained increase of serum creatinine to at least twice the lowest value observed during induction, or the need for longterm maintenance hemodialysis or transplantation. The primary and secondary endpoints were evaluated every 6 months for up to 7 years.

Statistical analysis. The proportions of patients who had either partial or complete response at 6 months were compared using a Pearson's chi-square test. The data were analyzed for all patients for whom outcome information was available. Differences in the baseline characteristics between the 2 groups and changes from baseline at each timepoint were evaluated with the use of the 2-sample $t$ test for continuous variables and the chi-square test for categorical variables. All variables that showed significant differences between the treatment groups $(\mathrm{p}<0.01)$ were subsequently analyzed using a logistic regression model. All p values were calculated as 2-sided.

Times to primary endpoint of renal flare were analyzed with the use of survival statistics. The cumulative survival curves were derived using the Kaplan-Meier method, and differences between the survival curves were compared using the log-rank method. In the analysis of renal survival, data were censored at the time of loss to followup.

\section{RESULTS}

Description of patients. Between January 2000 and February 2007, 261 biopsies consistent with a diagnosis of LN were identified in the NYUMC pathology database, corresponding to 237 patients with SLE. Females accounted for $82 \%$ of patients, and the mean age for these patients was $34.9 \pm 0.9$ years (range 5-78) at the time of the first biopsy. The biopsy results were as follows: 15 ISN/RPS class II, mesangial (6\%); 52 ISN/RPS class III, focal progressive glomerulonephritis (FPGN) (20.3\%); 106 ISN/RPS class IV, diffuse progressive glomerulonephritis (DPGN) (41\%); 68 ISN/RPS class V, membranous (MN) (27\%), and 15 ISN/RPS Class VI, sclerosis (6\%). There were 5 inconclusive reports.

Personal non-commercial use only. The Journal of Rheumatology Copyright @ 2009 . All rights reserved. 
Of the 237 patients, 161 were seen by physicians at NYUMC; biopsy specimens for the other 76 patients were sent from other medical centers for outside consultations. Records for 99 patients who were followed for at least 6 months after their biopsy were available and were reviewed.

Of the 99 patients, 85 were female $(86 \%)$ and 14 were male $(14 \%)$. The mean age at the time of the first biopsy was $34.2 \pm 1.1$ years (range 11-58). Most patients were from ethnic minorities (86\%): 24\% African American, 34\% Hispanic, and $28 \%$ Asian. In total, there were 125 biopsy results for these patients, corresponding to $22 \%$ FPGN, $40 \%$ DPGN, and 32\% membranous. Table 1 shows the characteristics of the SLE nephritis patients eligible for the study.

\section{Study outcome measures}

Induction of proliferative $L N$. Of the 77 cases of focal and diffuse proliferative nephritis (PN), $37(48 \%)$ were treated with CYC, 33 (43\%) were treated with MMF, and 7 did not receive immunosuppressive treatment. The baseline characteristics at the onset of treatment of both regimen groups, as detailed in Table 2, were similar. There were significant differences between the 2 groups with respect to the number of DPGN, serum creatinine, serum albumin, abnormal urinary sediment, prior use of MMF, and type of health insurance. A significantly greater number of patients with DPGN were treated with CYC $(78 \%)$ than with MMF $(55 \%)(\mathrm{p}=0.044)$. The average serum creatinine concentration was $1.5 \mathrm{mg} / \mathrm{dl}$ in the CYC group and $1.0 \mathrm{mg} / \mathrm{dl}$ in the MMF group (p = 0.009 ), and the average serum albumin was lower in the CYC group $(2.8 \mathrm{~g} / \mathrm{dl})$ compared to the MMF group (3.2 $\mathrm{g} / \mathrm{dl} ; \mathrm{p}=0.003) ; 87 \%$ of the CYC group had an abnormal urinary sediment compared to $64 \%$ of the MMF group ( $\mathrm{p}=$ 0.049). Fewer patients had prior CYC therapy in the CYC group versus the MMF group (18\% vs $31 \%$; $p=0.35$ ), but

Table 1. Baseline characteristics of the SLE patients eligible for analysis $(\mathrm{n}=99)$

\begin{tabular}{lc}
\hline Mean age, yrs (range) & $34.2 \pm 1.1(11-58)$ \\
Sex, F/M (\%) & $85 / 14(86 / 14)$ \\
Race, no. $(\%)$ & $13(14)$ \\
Caucasian & $22(24)$ \\
African American & $31(34)$ \\
Hispanic & $26(28)$ \\
Asian & 7 \\
Unknown & \\
Renal biopsy according to ISN/RPS class, no. $(\%)$ & $6(5)$ \\
II & $27(22)$ \\
III & $49(40)$ \\
IV & $39(32)$ \\
V & $2(2)$ \\
VI & 123 \\
Total & 2 \\
Inconclusive & \\
\hline
\end{tabular}

SLE: systemic lupus erythematosus; ISN/RPS: International Society of Nephrology/Renal Pathology Society. more had received MMF before (39\% vs $13 \%$; $\mathrm{p}=0.03$ ). Significantly more patients with private insurance were initially treated with MMF $(26 / 33,79 \%)$ versus CYC (18/37, $49 \% ; p=0.013)$. Complete or partial response was observed in 23 of the 33 (70\%) cases of PN treated with MMF, and 15 of the $37(41 \%)$ treated with CYC (Table 3).

Within the MMF group $(\mathrm{N}=33)$, nonresponders and responders were similar in age, sex, type of PN, activity and chronicity index, serum creatinine, serum albumin, abnormal complements, presence of anti-dsDNA antibodies, proteinuria, abnormal sediment, duration of disease, prior treatment with MMF or CYC, and type of health insurance. Statistically significant differences were noted with respect to race, specifically for Hispanics and Asians. All Asian patients treated with MMF responded $(\mathrm{N}=11)$, while clinical response was seen in only $45 \%$ of Hispanic patients (N $=5$ ). Hispanics accounted for $67 \%$ of nonresponders, while Asians accounted for $48 \%$ of responders ( $\mathrm{p}=0.04$ and 0.01 , respectively). There were no differences in response rates in patients with private insurance ( $70 \%$ vs $83 \%$; $\mathrm{p}=0.64$ ).

Within the CYC group $(\mathrm{N}=37)$ there were no significant differences between nonresponders and responders except for race. Similar to what was observed in the MMF group, there were more Hispanics (50\%) in the nonresponder group than in the responder group $(7 \%)(\mathrm{p}=0.01)$, with a response rate of only $9 \%$ ( 1 of 11 responded). The Asians comprised $40 \%$ of responders $(\mathrm{p}=0.27)$, with a response rate of $60 \%$ (6 of 10 responded). No difference was observed in the response of patients with private insurance $(\mathrm{p}=1.0)$.

Induction of membranous $L N$. Of the 39 cases of MN, 8 were treated with CYC, 15 with MMF, 4 with azathioprine, and 12 with prednisone alone. There were no significant differences between the CYC and MMF groups with respect to age, sex, race, laboratory abnormalities, duration of disease, or prior treatment with CYC or MMF. Serum creatinine and proteinuria were greater at baseline in the CYC group than in the MMF group, although not statistically significant. Table 4 summarizes the characteristics of these 23 cases. There were 10/15 (67\%) patients with private insurance treated with MMF and 2/8 (25\%) treated with CYC (p = $0.9)$. Similar to what was observed in PN, 11 of the 15 patients $(73 \%)$ treated with MMF had a complete or partial response compared to 3 of the 8 (38\%) treated with CYC. No significant differences between nonresponders and responders were observed (Table 5).

The overall response to induction for $\mathrm{PN}$ and $\mathrm{MN}$ was $34 / 48$ in the MMF group (71\%), and $18 / 45$ in the CYC group (40\%). All statistically different variables at $p<0.01$ were analyzed using a logistic regression model. Although only significant at the 0.05 level, nephritis class was included in the model. However, even after adjusting for race, serum creatinine, serum albumin, private insurance, and nephritis class, a logistic regression model showed that overall response to MMF was superior to that of CYC, with an

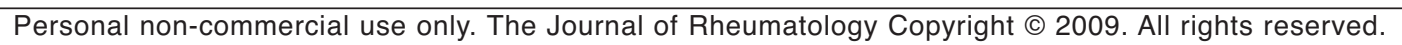


Table 2. Characteristics of the 70 proliferative nephritis cases by induction therapy. Values are no. (\%) or mean $\pm \mathrm{SD}$.

\begin{tabular}{|c|c|c|c|}
\hline & $\begin{array}{l}\text { MMF, } \\
\mathrm{N}=33\end{array}$ & $\begin{array}{c}\text { CYC, } \\
\mathrm{N}=37\end{array}$ & $\mathrm{p}$ \\
\hline Mean age, yrs & $33.2 \pm 1.8$ & $34.2 \pm 1.7$ & 0.88 \\
\hline Sex, female $(\%)$ & $30(91)$ & $30(81)$ & 0.32 \\
\hline \multicolumn{4}{|l|}{ Race, no. (\%) } \\
\hline Caucasian & $5(16)$ & $5(14)$ & 1.0 \\
\hline African American & $5(16)$ & $9(26)$ & 0.4 \\
\hline Hispanic & $11(34)$ & $11(31)$ & 1.0 \\
\hline Asians & $11(34)$ & $10(29)$ & 0.8 \\
\hline Unknown & 1 & 2 & - \\
\hline \multicolumn{4}{|c|}{ Renal biopsy according to ISN/RPS class, no. (\%) } \\
\hline III & $15(46)$ & $8(22)$ & $0.044^{\dagger}$ \\
\hline IV & $18(55)$ & $29(78)$ & \\
\hline Activity Index & $5.7 \pm 0.6(\mathrm{n}=30)$ & $7.8 \pm 0.8(\mathrm{n}=31)$ & 0.07 \\
\hline Chronicity Index & $2.6 \pm 0.5(\mathrm{n}=30)$ & $2.7 \pm 0.5(\mathrm{n}=31)$ & 0.93 \\
\hline Serum creatinine, $\mathrm{mg} / \mathrm{dl}$ & $1.0 \pm 0.07$ & $1.5 \pm 0.14$ & $0.009^{\dagger}$ \\
\hline Serum albumin, g/dl & $3.2 \pm 0.1$ & $2.8 \pm 0.1$ & $0.003^{\dagger}$ \\
\hline Abnormal complement, no. (\%) & $31(9)$ & $30(81)$ & 0.26 \\
\hline Presence of anti-dsDNA, no. (\%) & $27(82)$ & $31(84)$ & 1.00 \\
\hline Proteinuria, g/24 h & $3.4 \pm 0.55$ & $3.9 \pm 0.53$ & 0.55 \\
\hline Abnormal sediment, no. (\%) & $21(64)$ & $32(87)$ & $0.05^{\dagger}$ \\
\hline Duration of lupus nephritis, yrs & $3.7 \pm 0.8$ & $2.7 \pm 0.6$ & 0.55 \\
\hline Prior CYC treatment, no. (\%) & $8 / 26(31)$ & $5 / 28(18)$ & 0.35 \\
\hline Prior MMF treatment, no. (\%) & $3 / 26(12)$ & $11 / 28(39)$ & $0.03^{\dagger}$ \\
\hline \multicolumn{4}{|l|}{ Health insurance, no. (\%) } \\
\hline Service patient & $7(21)$ & $19(51)$ & $0.01^{\dagger}$ \\
\hline Private patient & $26(79)$ & $18(49)$ & \\
\hline
\end{tabular}

CYC: cyclophosphamide; MMF: mycophenolate mofetil; ISN/RPS: International Society of Nephrology/Renal Pathology Society. ${ }^{\dagger}$ Statistically different, $\mathrm{p}<0.05 . \pm$ Standard error of mean.

odds ratio $(\mathrm{OR})$ of 6.2 , but a very wide $95 \%$ confidence interval (CI) of 1.9-20.2 ( $\mathrm{p}=0.002)$. The only other significant predictor of response was being Hispanic: OR 0.17 (95\% CI 0.04-0.70, $\mathrm{p}=0.01$ ), Hispanics having worse outcome than Caucasians after adjusting for all the other variables.

Adverse events during induction. During this study period 5 deaths occurred: 1 that was likely related to the immunosuppressant effects of CYC (i.e., infection), 1 SLE related death in a patient receiving MMF, 1 due to SLE comorbidities and complications, and 2 deaths in patients that were treated only with steroids (no MMF or CYC) — one during cardiac surgery, the other a cardiac arrest.

Maintenance. Fifty-two patients responded to induction therapy ( 34 with MMF, 18 with CYC), 5 had just finished induction and were not included in this analysis. Of the remaining 47 patients (Table 6), 18 (38\%) had a subsequent flare and 29 remained flare-free after a mean followup of 2.5 \pm 0.2 years (range 1-5.5). Of these, 21 (72\%) had PN: 13 received MMF as induction and were followed for a mean of $2.7 \pm 0.4$ years, and 8 received CYC and were followed for a mean of $2.4 \pm 0.4$ years $(\mathrm{p}=\mathrm{NS})$.

During maintenance, most patients continued the treatment regimen that was assigned during induction. However,
5 patients with $\mathrm{PN}$ who responded to $\mathrm{CYC}$ were maintained on MMF; none of these patients had experienced flare after a mean followup of $2.1 \pm 0.4$ years.

There were $12(39 \%)$ flares in the MMF/MMF group (mean time to flare $1.8 \pm 0.3 \mathrm{yrs}$ ), and $6(38 \%)$ in the CYC/CYC group (mean time to flare $2.4 \pm 0.7 \mathrm{yrs} ; \mathrm{p}=1.0$ ), and one of these 6 coincided with an unplanned pregnancy. Of the $12 \mathrm{MMF} / \mathrm{MMF}$ flares, 8 occurred in PN (mean time to flare $1.9 \pm 0.3 \mathrm{yrs}$ ) and 4 in $\mathrm{MN}$ (time to flare $1.5 \pm 0.4$ yrs), while all the flares in the $\mathrm{CYC} / \mathrm{CYC}$ group occurred in patients with PN.

The maintenance of clinical response during longterm followup (up to 6 yrs) between these 3 treatments was compared using the log-rank method. Taking into account the time to recurrence and the different followup times, there was no statistical difference between the groups (log-rank $\mathrm{p}=0.26$ ). As noted during induction therapy, more patients with private insurance were maintained on MMF (27/31) than on CYC $(2 / 16)$.

There were 9 patients in the entire cohort who received chronic hemodialysis: 2 had a biopsy consistent with sclerosis, $6 \mathrm{PN}$, and $1 \mathrm{MN}$ (who did not receive therapy). Of the 6 PN, 1 presented with a serum creatinine of 5.3 and did not receive any treatment, 3 were initially treated with CYC

Personal non-commercial use only. The Journal of Rheumatology Copyright @ 2009 . All rights reserved. 
Table 3. Characteristics of the proliferative nephritis patients treated with $\mathrm{MMF}(\mathrm{N}=33)$ and $\mathrm{CYC}(\mathrm{N}=37)$ as induction therapy. Values are no. (\%) or mean \pm SD.

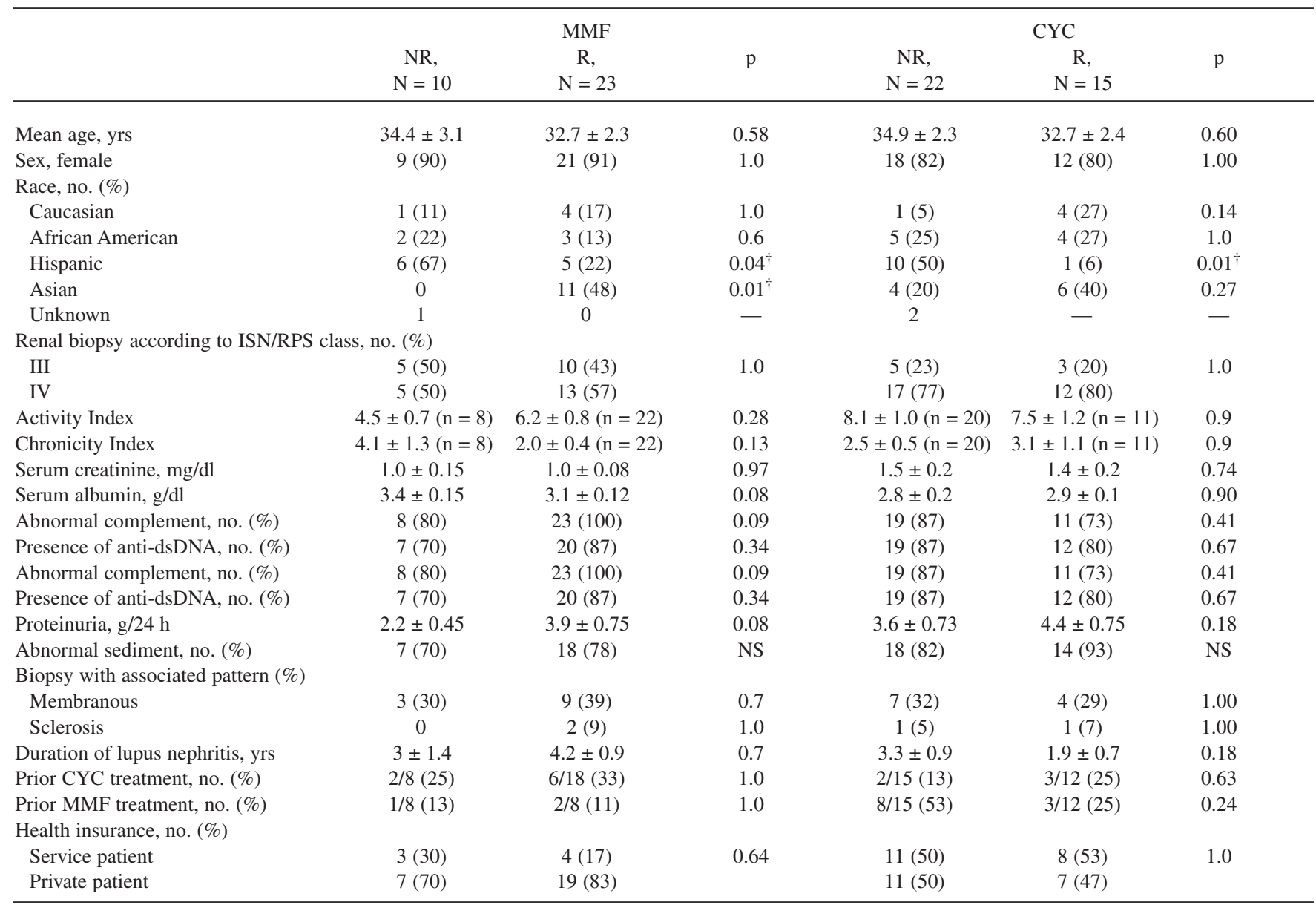

NR: nonresponder; R: responder; ISN/RPS: International Society of Nephrology/Renal Pathology Society; NS: nonsignificant; NA: nonapplicable.

$\dagger$ Statistically different; $\mathrm{p}<0.05 . \pm$ Standard error of mean.

with no response [one died soon after CYC was initiated, and another patient was rebiopsied (FPGN) and subsequently treated with $\mathrm{MMF} / \mathrm{MMF}$ with no response], and 2 received $\mathrm{MMF} / \mathrm{MMF}$ with no response (one of these was later unsuccessfully treated with $\mathrm{CYC} / \mathrm{CYC}$ ).

\section{DISCUSSION}

Clinical trials comparing the efficacy of different treatment regimens for $\mathrm{LN}$ have produced conflicting results due to the inherently complex nature of SLE. Accurate results are difficult to attain due to factors such as the incomplete understanding of the pathogenesis of SLE, the heterogeneous patient population and disease, the cyclical pattern of disease activity, and the concern for toxicities. It is for these reasons that the standard treatment for $\mathrm{LN}$, a combination of intravenous CYC and prednisone, has not changed since the 1980s. Nevertheless, the hope for a new standard is continually renewed through the emergence of newer, safer, and more effective immunosuppressants.

The most recent immunosuppressant medication, MMF, has already been studied by multiple groups, most notably by Chan, et $a l^{4,5}$ and Ginzler, et $a l^{6}$. A comparison of the results obtained by both groups reveals wide disparities that are likely attributed to differences in population and design. The patients in the Chan study were predominantly Asian, while the Ginzler study included a heterogeneous population, of which Asians represented 6\%. Other contributing factors may have been differences in the classes of nephritis included in the results (Chan: IV, Ginzler: III-V), and differences in the activity and chronicity indices of patients ${ }^{4-6}$.

Aspreva Pharmaceuticals released the preliminary results of a 2-phase study comparing MMF and CYC. After 24 weeks of therapy (185 patients), responses were similar to a response rate of $53 \%$ with CYC and $56 \%$ with $\mathrm{MMF}^{10}$.

Our results showed a greater rate of response with $\mathrm{MMF}$ (71\%) compared to CYC (40\%) during induction treatment, unlike the Aspreva data ${ }^{10}$, but similar to the data from Ginzler, et $a l^{6}$. The main reason for the discordance between our data and the Aspreva results may be prescribing trends by physicians. There was greater use of MMF in the private-

$$
\text { Personal non-commercial use only. The Journal of Rheumatology Copyright @ } 2009 \text {. All rights reserved. }
$$


Table 4. Characteristics of the 23 patients with membranous nephritis treated with mycophenolate mofetil (MMF) $(\mathrm{N}=15)$ and cyclophosphamide $(\mathrm{CYC})(\mathrm{N}=8)$ as induction therapy.

\begin{tabular}{|c|c|c|c|}
\hline & $\begin{array}{l}\text { MMF, } \\
\mathrm{N}=15\end{array}$ & $\begin{array}{l}\text { CYC, } \\
\mathrm{N}=8\end{array}$ & $\mathrm{p}$ \\
\hline Mean age, yrs & $32.3 \pm 1.4$ & $37.3 \pm 4.5$ & 0.17 \\
\hline Sex, female, n $(\%)$ & $13(87)$ & $7(88)$ & 1.00 \\
\hline \multicolumn{4}{|l|}{ Race, no. (\%) } \\
\hline Caucasian & $1(8)$ & $2(29)$ & 0.52 \\
\hline African American & $5(42)$ & $1(14)$ & 0.33 \\
\hline Hispanic & $3(25)$ & $1(14)$ & 1.00 \\
\hline Asian & $3(25)$ & $3(43)$ & 0.62 \\
\hline Unknown & 3 & 1 & \\
\hline Serum creatinine, $\mathrm{mg} / \mathrm{dl}$ & $0.98 \pm 0.1$ & $1.3 \pm 0.4$ & 0.72 \\
\hline Serum albumin, g/dl & $2.9 \pm 0.2$ & $2.8 \pm 0.1$ & 0.77 \\
\hline Abnormal complement, no. (\%) & $8(53)$ & $6(75)$ & 0.39 \\
\hline Presence of anti-dsDNA, no. (\%) & $6(40)$ & $6(75)$ & 0.19 \\
\hline Proteinuria, g/24 h & $3.9 \pm 0.65$ & $4.9 \pm 0.99$ & 0.53 \\
\hline Abnormal sediment, no. (\%) & 53.3 & 62.5 & NS \\
\hline Duration of lupus nephritis, yrs & $5.7 \pm 1.5$ & $2.9 \pm 0.9$ & 0.5 \\
\hline Prior CYC treatment, no. $(\%)$ & $3 / 12(25)$ & 2/7 (29) & NS \\
\hline Prior MMF treatment, no. $(\%)$ & $3 / 12(25)$ & $3 / 7(42)$ & \\
\hline \multicolumn{4}{|l|}{ Health insurance, no. (\%) } \\
\hline Service patient & $5(33)$ & $6(75)$ & 0.9 \\
\hline Private patient & $10(67)$ & $2(25)$ & \\
\hline
\end{tabular}

NS: nonsignificant. ${ }^{\dagger}$ Statistically different, $\mathrm{p}<0.05 . \pm$ Standard error of mean.

Table 5. Characteristics of the membranous nephritis treated with mycophenolate mofetil (MMF, N = 15) and cyclophosphamide $(\mathrm{CYC}, \mathrm{N}=8)$ as induction therapy.

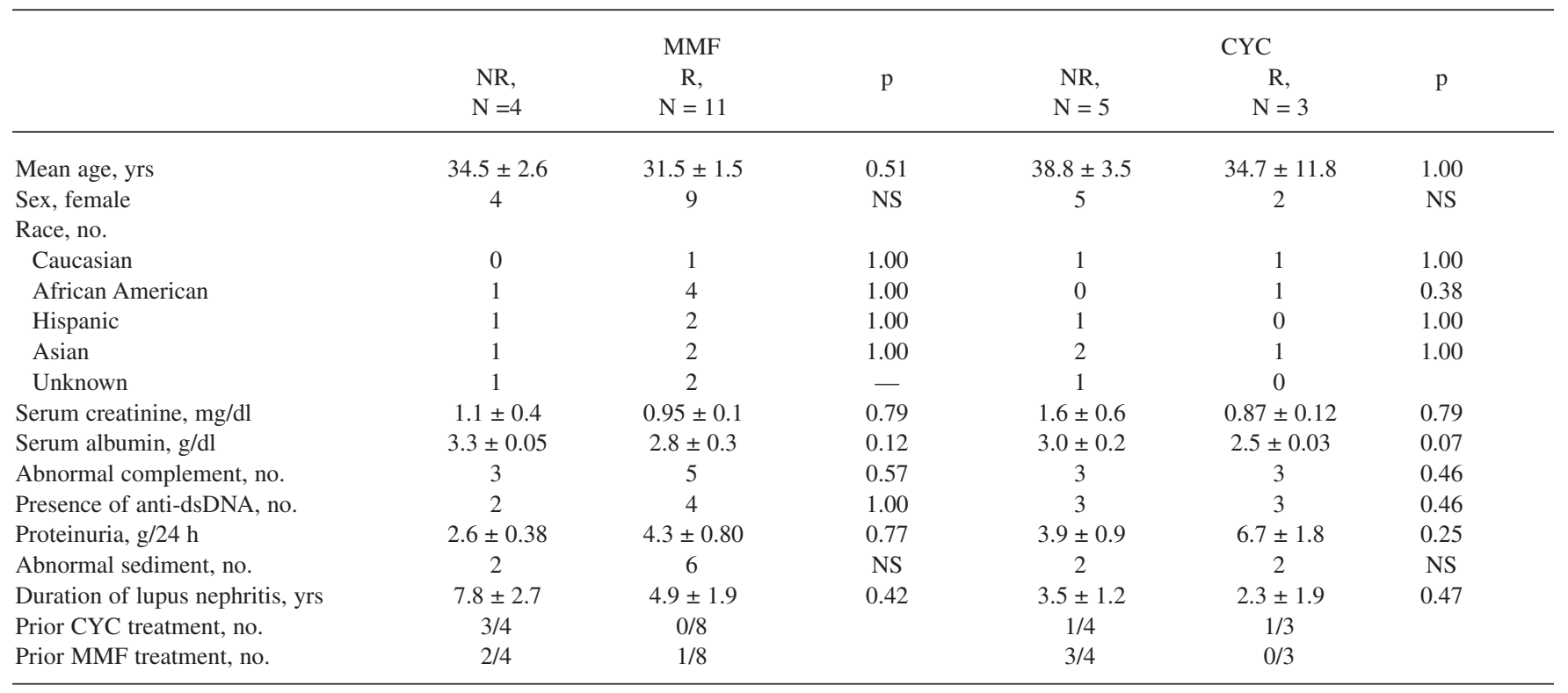

NR: nonresponder; R: responder; NS: nonsignificant; NA: nonapplicable. ${ }^{\dagger}$ Statistically different; $p$ value $<0.05 . \pm$ Standard error of mean.

practice setting; intravenous CYC was administered to patients unable to afford treatment with MMF. In addition, treatment with CYC was reserved for those patients who had failed MMF, or presented with poor prognostic characteristics, abnormal creatinine, scarring, or fibrosis ${ }^{1,14}$. The explanation for the difference between the Aspreva and the Ginzler data is likely the mandatory cross-over at 3 months in the Ginzler trial. It is possible that MMF facilitated a more rapid response than CYC, hence there were fewer "premature" cross-overs, or simply since this was an openlabel study the investigators were inclined to maintain patients on MMF a little longer than CYC, allowing MMF more time to be effective.

Our population was racially heterogeneous, and signifi- 


\begin{tabular}{|c|c|c|c|c|c|c|}
\hline & \multicolumn{3}{|c|}{ Proliferative } & \multicolumn{3}{|c|}{ Membranous } \\
\hline & $\begin{array}{l}\text { MMF, } \\
\mathrm{n}=21\end{array}$ & $\begin{array}{l}\text { CYC, } \\
\mathrm{n}=14\end{array}$ & $\mathrm{p}$ & $\begin{array}{l}\text { MMF, } \\
\mathrm{n}=10\end{array}$ & $\begin{array}{l}\text { CYC, } \\
\mathrm{n}=2\end{array}$ & $\mathrm{p}$ \\
\hline Flares, no. (\%) & $8(38)$ & $6(43)$ & 1.0 & $4(40)$ & 0 & 0.51 \\
\hline Time to flare, yrs & $1.9 \pm 0.3$ & $2.4 \pm 0.7$ & & $1.5 \pm 0.4$ & NA & \\
\hline No flares, no. (\%) & $13(62)$ & $8(57)$ & & $6(60)$ & 2 & \\
\hline Time of followup, yrs & $2.7 \pm 0.4$ & $2.4 \pm 0.4$ & & $2.3 \pm 0.3$ & $2.8 \pm 0.8$ & \\
\hline
\end{tabular}

CYC: cyclophosphamide; MMF: mycophenolate mofetil, NA: nonapplicable. All MMF flares (N=12) $1.8 \pm 0.3$.

cant trends were observed regarding race: all Asian patients responded to MMF, while $60 \%$ of the nonresponders in the MMF group were Hispanic. Our data for Hispanics receiving MMF is consistent with results from previous studies showing that these patients are less likely to respond favorably to $\mathrm{MMF}^{15}$. These results help reconcile the differences in responses between the Chan data and data from the US trials, including our own. Although the number of Asians in our cohort was small, their response rate to MMF was $100 \%$, consistent with the $95 \%$ response rate in Chan's study.

Finally, comparisons of MMF and CYC during maintenance therapy showed no statistical significance and were consistent with the results obtained in previous studies. Nevertheless, our data again demonstrated preferential use of MMF during maintenance, most likely due to its association with a lower toxicity. We fully acknowledge the limitation of a retrospective study (MMF groups were different compared to CYC groups).

The availability of new treatment regimens for LN, although none approved by the US Food and Drug Administration over the last 20 years, has caused an evolution in outcomes. Prior to use of CYC in nephritis, the present concepts of induction, remission, and maintenance did not exist. As demonstrated in the 1986 study comparing prednisone and intravenous CYC, the goal of treatment was solely to preserve a patient's life until endstage renal disease and death took their course $\mathrm{e}^{1,2,16}$. With the development of more effective medications and treatment regimens, primary outcome measures have acquired short and longterm components. The current goal of short-term therapy is improvement in abnormal laboratory and urine values within a period of 6 months or less, while the longterm goal remains the preservation of full renal function through immunosuppressive therapy. As a result of improved treatment regimens, $\mathrm{LN}$ no longer correlates directly to death by endstage renal disease.

Until new therapies become available, we must focus on improving the remission rates and side effects associated with current treatment options. The Euro-Lupus Nephritis Trial challenged the NIH protocol and concluded that lower and less toxic levels of CYC could be administered while maintaining the same response rate $^{17}$. Despite the com- pelling data presented by this group, higher levels of CYC continue to be administered in the United States, where the heterogeneity of the population raised concern about the ability to generalize the European data to US cohorts. Similar experiments might also help optimize treatment with MMF. Perhaps monitoring of mycophenolic acid levels in patients with LN, as performed in renal transplant patients, might help to improve the likelihood of renal remission. Further, studies comparing racial differences in the treatment of transplant patients with MMF have already concluded that African Americans typically require $3 \mathrm{~g}$ per day as compared to Caucasians, who typically require only $2 \mathrm{~g}$ per day ${ }^{18}$. Our results strongly suggest that a lot more information is needed with respect to race and ethnicity in the treatment of nephritis with MMF. Customized therapies are destined to play a role in the future of medicine, and it seems crucial for lupus to follow this trend given its variability. There is much to be done with the treatments available to us now; optimized treatments will most likely improve outcomes and quality of life for our patients.

\section{REFERENCES}

1. Austin HA, Klippel JH, Balow JE, et al. Therapy of lupus nephritis. Controlled trial of prednisone and cytotoxic drugs. N Engl J Med 1986;314:614-9.

2. Steinberg AD, Steinberg SC. Long-term preservation of renal function in patients with lupus nephritis receiving treatment that includes cyclophosphamide versus those treated with prednisone only. Arthritis Rheum 1991;34:945-50.

3. Contreras G, Pardo V, Leclercq B, et al. Sequential therapies for proliferative lupus nephritis. N Engl J Med 2004;350:971-80.

4. Chan TM, Li FK, Tang CS, et al. Efficacy of mycophenolate mofetil in patients with diffuse proliferative lupus nephritis. Hong Kong-Guangzhou Nephrology Study Group. N Engl J Med 2000;343:1156-62.

5. Chan TM, Tse KC, Tang CS, Mok MY, Li FK; Hong Kong Nephrology Study Group. Long-term study of mycophenolate mofetil as continuous induction and maintenance treatment for diffuse proliferative lupus nephritis. J Am Soc Nephrol 2005;16:1076-84.

6. Ginzler EM, Dooley MA, Aranow C, et al. Mycophenolate mofetil or intravenous cyclophosphamide for lupus nephritis. N Engl J Med 2005;353:2219-28.

7. Zhu B, Chen N, Lin Y, et al. Mycophenolate mofetil in induction and maintenance therapy of severe lupus nephritis: a meta-analysis of randomized controlled trials. Nephrol Dial Transplant 2007;22:1933-42 
8. Allison AC, Eugui EM. Mycophenolate mofetil and its mechanisms of action [review]. Immunopharmacology 2000;47:85-118.

9. Moore RA, Derry S. Systematic review and meta-analysis of randomised trials and cohort studies of mycophenolate mofetil in lupus nephritis [review]. Arthritis Res Ther 2006;8:R182.

10. Aspreva and Roche announce preliminary results for phase III study evaluating CellCept in LN. [Internet. Accessed October 10, 2008.] Available from: http://www.aspreva.com/completenews.php?id=136

11. Fernandez M, Calvo-Alén J, Alarcón GS, et al. Systemic lupus erythematosus in a multiethnic US cohort (LUMINA): XXI. Disease activity, damage accrual, and vascular events in pre- and postmenopausal women. Arthritis Rheum 2005;52:1655-64.

12. Weening JJ, D'Agati VD, Schwartz MM, et al. The classification of glomerulonephritis in systemic lupus erythematosus revisited. Kidney Int 2004;65:521-30.

13. Austin HA III, Klippel JH, Balow JE, et al. Therapy of lupus nephritis: controlled trial of prednisone and cytotoxic drugs. N Engl J Med 1986;314:614-9.
14. Illei GG, Takada K, Parkin D, et al. Renal flares are common in patients with severe proliferative lupus nephritis treated with pulse immunosuppressive therapy: long-term followup of a cohort of 145 patients participating in randomized controlled studies. Transplantation 1998;65:242-8.

15. Contreras G, Lenz O, Pardo V, et al. Outcomes in African Americans and Hispanics with lupus nephritis. Kidney Int 2006;69:1846-51

16. Gourley MF, Austin HA III, Scott D, et al. Methylprednisolone and cyclophosphamide, alone or in combination, in patients with lupus nephritis. A randomized, controlled trial. Ann Intern Med 1996;125:549-57.

17. Houssiau FA, Vasconcelos C, D'Cruz D, et al. Early response to immunosuppressive therapy predicts good renal outcome in lupus nephritis: lessons from long-term followup of patients in the Euro-Lupus Nephritis Trial. Arthritis Rheum 2004;50:3934-40.

18. Schweitzer EJ, Yoon S, Fink J, et al. Mycophenolate mofetil reduces the risk of acute rejection less in African-American than in Caucasian kidney recipients. Kidney Int 2006;69:1846-51. 